Original Research Paper

\title{
New World Mucocutaneous Leishmaniasis with Oral Manifestations: Case Report and Damage Repair
}

\author{
${ }^{1}$ Manuel Antonio Gordón-Núñez, ${ }^{2}$ Stefânia Jeronimo Ferreira, ${ }^{2}$ Ana Luiza Dias Leite de Andrade, ${ }^{3}$ Kléber \\ Giovanni Luz, ${ }^{3}$ Eveline Pipolo Milan and ${ }^{2}$ Hébel Cavalcanti Galvão \\ ${ }^{1}$ Department of Dentistry, State University of Paraiba, Araruna, PB, Brazil \\ ${ }^{2}$ Department of Dentistry, Federal University of Rio Grande do Norte, Natal, RN, Brazil \\ ${ }^{3}$ Division of Infectology, Giselda Trigueiro Hospital, Federal University of Rio Grande do Norte, Natal, RN, Brazil
}

\author{
Article history \\ Received: 02-10-2014 \\ Revised: 07-01-2015 \\ Accepted: 31-01-2015 \\ Corresponding Author: \\ Ana Luiza Dias Leite de Andrade \\ Departamento de Odontologia \\ Av. Senador Salgado Filho, \\ Universidade Federal do Rio \\ Grande do Norte, \\ 1787-Lagoa Nova-Natal-RN- \\ Brasil \\ Email: ana_luiza_dla@hotmail.com
}

\begin{abstract}
New World cutaneous leishmaniasis is an infectious and parasitic disease caused by protozoan species of the genus Leishmania. The disease is transmitted by the biteoffemaleinsects of the genus Lutzomyia and is endemic in some countries of the New World, including Bolivia, Peru and Brazil. The diagnosis of New World mucocutaneous leishmaniasis encompasses epidemiological, clinical and laboratory aspects. Usually, the combination of some of these elements is necessary for the final diagnosis. Oral lesions of leishmaniasis can be challenging to diagnose and represent a complication to the overall health of the individual. We report the case of a 28 -year-old man who presented multiple lesions in the soft palate, upper and lower lips. So, we highlight the important role of the dentist in the diagnosis of oral leishmaniasis lesions, which are uncommon and can be confused with other diseases, thus contributing to the identification and appropriate treatment of cases, especially in geographical regions where the parasite is endemic.
\end{abstract}

Keywords: Cutaneous Leishmaniasis, Lutzomya, Mucous Leishmaniasis, Oral Mucosa

\section{Introduction}

New World Cutaneous Leishmaniasis is an infectious and parasitic disease caused by protozoan species of the genus Leishmania, such as Leishmania (Viannia) braziliensis, L. (V) guyanensis and $L$. (V) panamensi (Hernández, 2006; Von Stebut, 2007). The disease is transmitted by the bite of female insects of the genus Lutzomyia and the leishmania promastigotes are introduced into the skin when feeding on the blood of a vertebrate host (Von Stebut, 2007; Mitropoulos et al., 2010; De Paulo et al., 2013). The promastigotes invade the cells of the monocyte phagocytic system, transform into amastigotes, multiply within phagolysosomes and invade other cells (Hernández, 2006; Von Stebut, 2007; Mitropoulos et al., 2010; De Paulo et al., 2013).

Cutaneous Leishmaniasis is endemic in some countries of the New World, including Bolivia, Peru and Brazil. In the last two decades, there has been a significant increase in the number of cases of such infection in almost all states of Brazil, probably associated with deforestation actions (Mitropoulos et al., 2010; Gontijo and Carvalho, 2003).

In New World cutaneous leishmaniasis, the amastigote incubation period is usually 10 days to 3 months until the appearance of clinical signs of infection. Commonly, the primary disease manifests as a single papular lesion on the site of the insect bite, which increases in size and often become ulcerated (Mitropoulos et al., 2010; Gontijo and Carvalho, 2003). On the other hand, multiple insect bites or local spread may generate a greater number of lesions, which with the evolution of the pathological course of the disease, may acquire different presentations, such as impetigoid, tuberculoid and nodular vegetative forms as well as indurated ulcers with elevated borders in a gross granulation tissue background, giving the classic appearance of "border in frame". A period of 3 to 18 months is sufficient for the repair of these lesions by more than $90 \%$ of cases (Salman et al., 
1999; Gontijo and Carvalho, 2003; Silveira et al., 2004; Piscopo and Mallia, 2006).

For mucosal leishmaniasis, the incubation period ranges from 3 to 18 months, but the manifestation of the disease may occur months or years after the repair of cutaneous lesions and early cases of simultaneous involvement of skin and mucous membranes has been reported (Hernández, 2006; Motta et al., 2007; Marra et al., 2014). The mucosal involvement by Leishmania is unusual and results from hematogenous or lymphatic dissemination of amastigotes derived from the skin to the naso-oropharyngeal mucosa. Generally, this process causes adestructive infectious-inflammatory reaction and oral lesions give rise to ulcers in more than $5 \%$ of cases of leishmaniasis with a history of previous cutaneous lesions, especially involving the hard and soft palate. However, the disease can compromise anywhere in the mouth as exophytic, nodular and induratedlesions (Costa Jr et al., 2003; Van Damme et al., 2004; De Paulo et al., 2013; Marra et al., 2014).

The diagnosis of New World mucocutaneous leishmaniasis encompasses epidemiological, clinical and laboratory aspects. Usually, the combination of some of these elements is necessary for the final diagnosis (Motta et al., 2007; De Paulo et al., 2013). The treatment of cutaneous leishmaniasis can accelerate healing and reduces scar formation, which is particularly important for aesthetic visible regions of the body, while also reducing the risk of mucosal involvement. A local or systemic therapy may be applied and, traditionally, the most used drugs are pentavalent antimonial compounds. Alternate treatments include amphotericin $\mathrm{B}$, oral ketoconazole and interferon gamma. In addition to drug therapy, adequatenutrition must be provided for the patient and the need for possible ventilatory support assessed (Virgilio and Hale, 2004; Tiuman et al., 2011).

The aim of this article was to report a case of New World mucocutaneous leishmaniasis with severe oral manifestations and discuss the main aspects related to the disease.

\section{Case Report}

A 28-year-old immune competent man was seen at the Giselda Trigueiro Hospital (Natal/RN, Brazil) with multiple lesions in the oral cavity for about 1 year. Medical record had history of abundant overlying purulent discharge affecting upper lip. In the latter location, it was observed swelling and bleeding points. The patient complained of an ulcerated lesion on the dorsum of the right foot with raised edges, which had emerged 10 months before. The clinical evidences and laboratorial analysis excluded the diagnostic hypotheses of tuberculosis and retroviral infection. The patient resided in Belem-PA, a Northern Brazilian city, six years ago and remained in that region for about two years.

Due to the multiples oral lesion, observed in the first evaluation by another medical team, incisional biopsy was performed and histopathologic diagnosis was moderately differentiated squamous cell carcinoma. The patient was subjected to 5 cycles of chemotherapy, followed by partial improvement of the lesion. About a year after, the biopsy of the lesion on the right foot was performed, which was suggestive of fungal microorganism with single sporulation, resulting in the diagnosis of paracoccidioidomycosis. The patient was hospitalized for treating paracoccidioidomycosis with amphotericin B (total dose of about $1000 \mathrm{mg}$ ) followed by maintenance therapy with sulfamethoxazole and Trimethoprim $(800+160 \mathrm{mg}$ two times daily), but he did not cooperate with treatment again. Because of this, there were episodes of exacerbations of vegetative lesions located in soft palate and upper lip associated with secondary infection, which promoted five hospitalizations with prescription of amphotericin B and antibiotics.

During this period, the patient reported that he had realized a biopsy on the left arm three years before and the diagnosis was cutaneous leishmaniasis. At this moment, the treatment used was based on the application of intramuscular injection of antimoniate of N-methyl glucamine (Glucantime ${ }^{\circledR}$-09ampoules). However, the treatment was not completed due to lack of patient compliance.

Ten years after the biopsy on the arm, although the patient claimed he would have continued the use of sulfamethoxazole and Trimethoprim, he was hospitalized due to worsening of oral lesions, since beyond the palate and upper lip, the lower lip was also compromised. At this time, the patient was referred for oral evaluation by our staff and intraoral clinical examination showed multipleulcerative lesions with granulomatous aspect with extension to the hard palate and plentiful crusty lesions affecting the lips covered with purulent exudate and areas of observed swelling and bleeding points (Fig. 1A, B). The patient related weight loss (6 pounds), dysphonia, dysphagia. He was under treatment with amphotericin B deoxycholate.

Aiming to document the case with teaching purposes, signed informed consent was obtained from the patient and a new incisional biopsy of lesions in soft palate and upper lip was performed. The specimens were analyzed at the Anatomical Pathology Service, Department of Dentistry, Federal University of Rio Grande do Norte. 


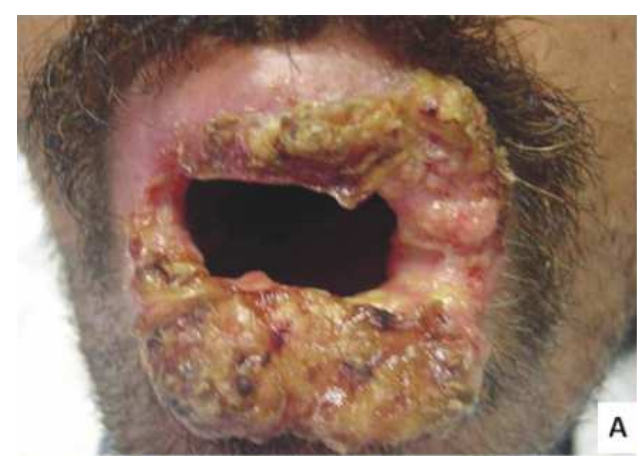

(a)

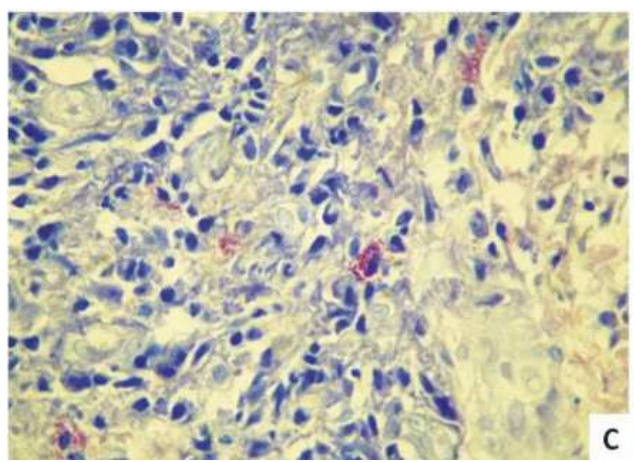

(c)

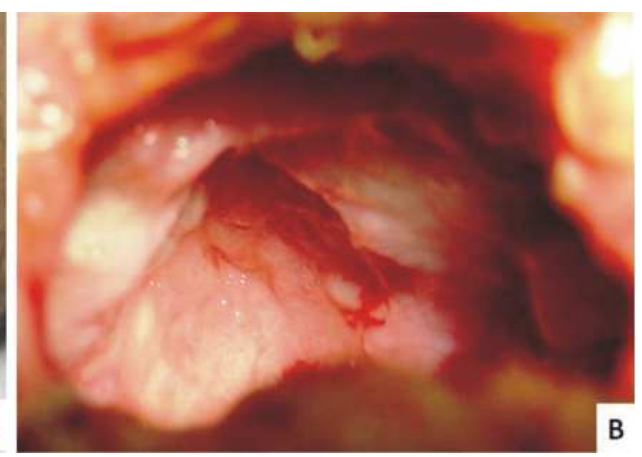

(b)

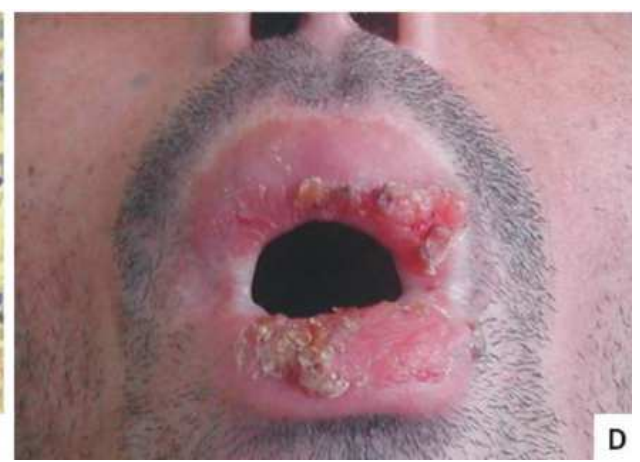

(d)

Fig. 1. (A), Extra-oral lesions with vegetating appearance and areas of crusting formation on the lips. (B), Intraoral aspect of granulomatous lesions on the palate. (C), Histiocytes containing amastigotes of Leishmania (red circles). Giemsa staining, 100×. (D), Patient showingregression of lip lesions and microstomia two months after treatment

Histopathological analysis of slides stained with hematoxylin-eosin and visualized by light microscopy revealed the presence of a dense granulation reaction consists of a mixed inflammatory infiltrate. The slides were prepared with Giemsa stain, which showed histiocytes containing numerous intracytoplasmic rounded bodies compatible with amastigotes of Leishmania (Fig. 1C). On the basis of this evidences, histopathological diagnosis of Mucocutaneous leishmaniasis was established.

Aiming to confirm the histopathological diagnosis, serology for leishmaniasis was performed by indirect immunofluorescence, which was positive with a titer of 1:80. Montenegro's intradermal reaction was also positive with induration of $11 \mathrm{~mm}$ diameter and it was realized the direct microscopic demonstration of the parasite from the material collected from the lesions. The patient was treated with Glucantime ${ }^{\circledR}$ for 30 days, which resulted in complete regression of the lesions on the palate and lips (Fig. 1D) the dysphonia and dysphagia were totally solved during the medicamentous treatment, but due to scarring of the labial lesions the development of microstomia occurred. The patient was referred for reconstructive plastic surgery and is currently free of oral and cutaneous lesions, but the microstomia was not totally solved.

\section{Discussion}

Leishmaniasis Occurs in different areas of the world and is considered endemic in 88 countries (Aliaga et al., 2003; Motta et al., 2007; De Paulo et al., 2013). Three main clinical forms of the disease are known: Visceral, cutaneous and mucous leishmaniasis (Hernández, 2006; Piscopo and Mallia, 2006). It is reported that all known pathogenic Leishmania species are capable of causing skin and/or mucosa clinical disease in humans (Aliaga et al., 2003; Motta et al., 2007).

In New Worldcountries which Mucocutaneous leishmaniasisis endemic, the disease has become a public health problem (Mitropoulos et al., 2010). In Brazil, the prevalence of cutaneous leishmaniasis is the largest in the Americas, with an annual average of 35,000 cases (Velozo et al., 2006). The importance of the disease is not only in its high incidence and wide geographical distribution, but also in the possibility of having presentations that may determine destructive, disfiguring 
and disabling injuries, with major repercussions in psychosocial aspect of the individual (Costa Jr et al., 2003; Gontijo and Carvalho, 2003; Piscopo and Mallia, 2006). Because of the length and severity of the lesions of the mouth, the patient of the present case reported dysphagia and dysphonia, impairment of mouth opening and withdrawn behavior due to the clinical appearance of the lesions on his lips.

In endemic areas, the protozoan can persist as a latent parasite for a long period of time and under conditions of immunosuppression, the disease can manifest (Ferreli et al., 2004; Van Damme et al., 2004; Von Stebut, 2007). The New World cutaneous leishmaniasis can display a variety of clinical manifestations resulting from the replication of the parasite in macrophages (Piscopo and Mallia, 2006; Von Stebut, 2007). Generally, the lesions become clinically observable within a few weeks of amastigotes incubation, starting with single papule or nodule, which ulcerate centrally and later can exhibit hardened high edges (border frame lesion) (Lawn et al., 2004; Motta et al., 2007).

Cutaneous infection affects exposed areas of the body and appears inpatients from endemic areas or who have been in such regions recently (Gontijo and Carvalho, 2003). In the present case, the patient had a history of two years of residence in a city considered a risk area for the disease (Belem-PA, Brazil), which may have been the site of infection acquisition. Furthermore, the cutaneous lesions occurred in the arm and foot and the clinical appearance of them was not different from that reportedin the literature (Salman et al., 1999; Motta et al., 2007).

A wide variety of clinical suspicion may contribute to delay in diagnosis of skin infection, which facilitates the spread of the parasite through hematogenous and/or lymphatic route, increasing the risk of installation of amastigotes in the nasooropharyngeal mucosa and the development of mucous leishmaniasis (Lambertucci et al., 2003; Nakkash-Chmaisse et al., 2011; Marra et al., 2014). It is noteworthy that this process is compounded by the institution of a treatment in time and manner inadequate. Probably, in the case reported here, no specific therapy for Leishmania described in the clinical history may have played an important role in the persistence of lesions in skin and oral mucosa. Thus, this case corroborates the literature reports on the difficulty of diagnosing skin lesions of leishmaniasis (Costa Jr et al., 2003; Gontijo and Carvalho, 2003; Motta et al., 2007).

Mucosal lesions generally occur months or even years after primary repair of skin lesions, but both presentation scan manifest concomitantly (Costa Jr et al., 2003; De Paulo et al., 2013; Marra et al., 2014). It is important to mention that even rare, primary mucosal lesions can occur in both immunocompromised and immunocompetent patients (Madeddu et al., 2014). In the present case, oral lesions occurred after the appearance of skin lesions and were important to establish the differential diagnosis of the disease.

It is believed that mucosal lesions seem to be more the result of immune-mediated reactions than due the toxic effect of the parasite. However, the biological immune evasion mechanism that allows the initial spread of the parasite and the subsequent establishment of an immune-mediated disease is little known (Silveira et al., 2004). The lesions of New World mucosal leishmaniasis mainly undertake the upper respiratory tract, with predilection for the nose and larynx (Velozo et al., 2006; Marra et al., 2014).

Similarly with cutaneous form, the lesions of mucosal leishmaniasis exhibit several clinical presentations and can make differential diagnosis with diseases including syphilis, tuberculosis, squamous cell carcinoma and other malignancies, paracoccidioidomycosis, histoplasmosis and sarcoidosis, among others (Lawn et al., 2004; Virgilio and Hale, 2004; Motta et al., 2007). In the case reported here, this difficulty was found in establishing the clinical diagnosis of oral lesions, which can be verified by the hypothesesof tuberculosis and retro virosis initially suspected.

Intraorally, a higher frequency of lesions in the palate has been reported in the literature (Aliaga et al., 2003; Van Damme et al., 2004; Motta et al., 2007). In this case, the lesions seem to have started by the involvement of the upper lip, with subsequent extension to the lower lip and progression to severe impairment of these tissues. Thus, the commitment of the lips by infection is uncommon, which makes the case reported here quite interesting (Ferreli et al., 2004; Motta et al., 2007).

The clinical appearance of the lesions of leishmaniasis in mouth not follow a particular pattern, ranging from small ulcerations to massive swellings, sometimes with vegetating presentation, suggesting an extensive range of diagnostic hypotheses of infectious or neoplastic character (Costa Jr et al., 2003; Motta et al., 2007). In the case reported here, the patient developed lesions that apparently influenced the different clinical diagnoses, which included tuberculosis, retrovirosis and squamous cell carcinoma.

The diagnosis of New World cutaneous and mucosal leishmaniasis covers epidemiological, clinical and laboratory aspects. Generally, the combination of 
some of these elements is necessary to establish the final diagnosis (Mitropoulos et al., 2010). The New World cutaneous leishmaniasis comprises a variety of clinical manifestations that depend on factors, such as the species of Leishmania involved and the relationship between the parasite and the host (Piscopo and Mallia, 2006).

Laboratory diagnosis of cutaneous and mucosal forms of leishmaniasis is based on search of the parasite in infected tissues by direct demonstration in microscopy or the disclosure of amastigotes within macrophages by Giemsa stain in tissue samples obtained from biopsy (Madeddu et al., 2014). Importantly, the latter method does not provide specific diagnostic information, since other infections can present the same histopathology of intracytoplasmic inclusions in histiocytes (Costa Jr et al., 2003).

The direct demonstration in microscopy realized from material collected from skin crusts or biopsied specimens, is a quick and inexpensive technique, but that has limited sensitivity, especially in chronic lesions (Piscopo and Mallia, 2006).

Other diagnostic techniques include the culture of the parasite and indirect methods including the Montenegro skin test, serology for Leishmania-specific antibodies, such as Enzyme-Linked Immuno Sorbent Assay (ELISA) and, more recently, the detection of DNA of Leishmania by techniques of molecular biology, such as Polymerase Chain Reaction (PCR) (Mitropoulos et al., 2010; Nakkash-Chmaisse et al., 2011; Faber et al., 2003).

The culture material collected from the lesions of leishmaniasis, although very sensitive, can be contaminated by bacteria or fungi in blood sampling or sample handling. The Montenegro skin test detects specific cutaneous hypersensitivity, but its limitation liesin the inability to differentiate a current and a previous infection, besides the risk of false positive due to cross-reactions with other cutaneous infections (Faber et al., 2003; Piscopo and Mallia, 2006). On the other hand, negative results in Montenegro skin test could be useful to exclude leishmaniasis in those suspected cases living in endemic areas. The PCR displays considerable therapeutic and prognostic implications, because it offers higher sensitivity and specificity in the diagnosis of the disease and provide information onthe identification of Leishmania species associated with infection (Mitropoulos et al., 2010). However, these reactions generally either amplify genusand subgenus-specific conserved regions or require separate primer pairs for each Leishmania species, promoting an unacceptable cost to the patient attended by the public health system and importance to use this technique with scientific research interest or in populations with financial resources to enable you to choose, if necessary, use this more specific diagnostic technique (Disch et al., 2005; Bensoussan et al., 2006).

The reported literature shows that, even with the clinical information and the results of the histopathological examination, the diagnosis of lesions in the mouth may be difficult (Motta et al., 2007). In the case reported here, the diagnosis was established with complementary results from the Montenegro skin test and Giemsa stain, which were all positive.

Treatment of cutaneous leishmaniasis can accelerate healing and reduce scar formation, which is very important in a esthetically visible regions of the body (Mitropoulos et al., 2010; Reveiz et al., 2013). Therapy maybe local or systemic and is usually performed with the use of drugs, such as amphotericin B, liposomal amphotericin B and Glucan time ${ }^{\circledR}$ (Reveiz et al., 2013). Systemic treatment is important because it promotes the repair of lesions and limits the risk of developing mucosal disease (Virgilio and Hale, 2004; Von Stebut, 2007; Reveiz et al., 2013). It has been reported that untreated mucosal leishmaniasis do not heal spontaneously and cause crippling destruction of affected tissues (Costa Jr et al., 2003). In the case reported here, the lack of establishment of early treatment contributed to the development of severe lesions in the mouth.

Due to the severity of mucosal lesions with destruction or deformation of tissues, when it is possible, is part of the therapeutic management the performing of plastic surgery (Velozo et al., 2006). Because of the presence of microstomia and deformation of the lips, our patient was referred for reconstructive plastic surgery. In addition to improving facial aesthetics, this conduct has a positive effect on self-esteem of individual who feels stigmatized by injuries.

This case clearly demonstrates that the mucocutaneous leishmaniasis is a debilitating disease that can lead directly or indirectly to compromised immune system, increasing the risk of secondary infections. Oral lesions can be challenging to diagnose and represent a complication to the overall health of the individual. Therefore, based on a case report and literature review, we highlight the important role of the dentist in the diagnosis of oral leishmaniasis lesions, which are uncommon and can be confused with other diseases, thus contributing in the identification and appropriate treatment of cases, especially in geographical regions where the parasite is endemic. 


\section{Funding Information}

The authors have no support or funding to report.

\section{Author's Contributions}

All authors equally contributed in this work.

\section{Ethics}

This article is original and contains unpublished material. The corresponding author confirms that all of the other authors have read and approved the manuscript and no ethical issues involved.

\section{References}

Aliaga, L., F. Cobo, J.D. Mediavilla, J. Bravo and A. Osuna et al., 2003. Localized mucosal leishmaniasis due to Leishmania (Leishmania) infantum: Clinical and microbiologic findings in 31 patients. Med. (Baltimore), 82: 147-158. PMID: 12792301

Bensoussan, E., A. Nasereddin, F. Jonas, L.F. Schnur and C.L. Jaffe, 2006. Comparison of PCR assays for diagnosis of cutaneous leishmaniasis. J. Clin. Microbiol., 44: 1435-1439.

DOI: 10.1128/JCM.44.4.1435-1439.2006

Costa Jr, J.W., D.A. Milner Jr and J.H. Maguire, 2003. Mucocutaneous leishmaniasis in a US citizen. Oral Surg., Oral Med. Oral Pathol., Oral Radiol. Endodontol., 96: 573-577. DOI: 10.1016/S1079-2104(03)00299-3

De Paulo, L.F., G.F. Rocha, C.M. LuisiJr, R.R. Rosa and A.F. Durighetto Jr, 2013. Mucocutaneous leishmaniasis: Mucosal manifestations in an endemic country. Int. J. Infect. Dis., 17: 1088-1089. DOI: $10.1016 /$ j.ijid.2013.05.013.

Disch, J., M.J. Pedras, M. Orsini, C. Pirmezand M.C. De Oliveira et al., 2005. Leishmania (Viannia) subgenus kDNA amplification for the diagnosis of mucosal leishmaniasis. Diagnostic Microbiol. Infect. Dis., 51: 185-190.

DOI: 10.1016/j.diagmicrobio.2004.10.005

Faber, W.R., L. Oskam, T. Van Gool, N.C. Kroon and K.J. Knegt-Junk et al., 2003. Value of diagnostic techniques for cutaneous leishmaniasis. J. Am. Acad. Dermatol., 49: 70-74. DOI: 10.1067/mjd.2003.492

Ferreli, C., L. Atzori, M. Zucca, P. Pistis and N. Aste, 2004. Leishmaniasis of the lip in a patient with Down's syndrome. J. Eur. Acad. Dermatol. Venereol., 18: 599-602. DOI: 10.1111/j.1468-3083.2004.00987.x

Gontijo, B. And M.L.R. Carvalho, 2003. Leishmaniose tegument Aramericana. Rev. Soc. Bras. Med. Trop., 36: 71-80.
Hernández, C.A., 2006. Natural history of cutaneous and mucocutaneous leishmaniasis. Biomedica. Suppl., 1: 10-12. PMID: 17361836

Lambertucci, J.R., R. Coulaud and L.C.S. Silva, 2003. Mucosal Leishmaniasis. Rev. Soc. Bras. Med. Trop., 36: 307-308.

Lawn, S.D., J. Whetham, P.L. Chiodini, J. Kanagalingam and J. Watson et al., 2004. New world mucosal and cutaneous leishmaniasis: An emerging health problem among British travellers. QJM, 97: 781-788. DOI: 10.1093/qjmed/hch127

Madeddu, G., M.L. Fiori, P. Ena, F. Riu and C. Lovigu et al., 2014. Mucocutaneous leishmaniasis as presentation of HIV infection in Sardinia, insular Italy. Parasitol. Int., 63: 35-36.

DOI: $10.1016 /$ j.parint.2013.10.002

Marra, F., M.C. Chiappetta and V. Vincenti, 2014. Ear, nose and throat manifestations of mucocutaneous leishmaniasis: A literature review. Acta Biomed., 85: 3-7. PMID: 24897964

Mitropoulos, P., P. Konidas and M. Durkin-Konidas, 2010. New World cutaneous leishmaniasis: Updated review of current and future diagnosis and treatment. J. Am. Acad. Dermatol., 63: 309-322. DOI: 10.1016/j.jaad.2009.06.088.

Motta, A.C., M.A. Lopes, F.A. Ito, R. Carlos-Bregni and O.P. De Almeida et al., 2007. Oral leishmaniasis: A clinicopathological study of 11 cases. Oral Dis., 13: 335-340. DOI: 10.1111/j.1601-0825.2006.01296.x

Nakkash-Chmaisse, H., R. Makki, G. Nahhas, K. Knio and N. Nuwayri-Salti, 2011. Detection of Leishmania parasites in the blood of patients with isolated Cutaneous Leishmaniasis. Int. J. Infect. Dis., 15: 491-494. DOI: 10.1016/j.ijid.2011.03.022

Piscopo, T.V. and C.M. Azzopardi, 2006. Leishmaniasis. Postgrad. Med. J., 82: 649-657. DOI: $10.1136 /$ pgmj.2006.047340

Reveiz, L., A.N. Maia-Elkhoury, R.S. Nicholls, G.A Romero and Z.E. Yadon, 2013. Interventions for American cutaneous and mucocutaneous leishmaniasis: A systematic review update. PLoS One. DOI: 10.1371/journal.pone.0061843

Salman, S.M., N.G. Rubeiz and A.G. Kibbi, 1999. Cutaneous leishmaniasis: Clinical features and diagnosis. Clin. Dermatol., 17: 291-296. PMID: 10384868

Silveira, F.T., R. Lainson and C.E. Corbett, 2004. Clinical and immunopathological spectrum of American cutaneous leishmaniasis with special reference to the disease in Amazonian Brazil: A review. Men Inst. Oswaldo. Cruz., 99: 239-251. PMID: 15273794 
Tiuman, T.S., A.O. Santos, T. Ueda-Nakamura, B.P. Filho and C.V. Nakamura, 2011. Recent advances in Leishmaniasis treatment. Int. J. Infect. Dis., 15: 525-532. DOI: 10.1016/j.ijid.2011.03.021

Van Damme, P.A., M. Keuter, S. Van Assen, P.C. DeWilde and P.J. Beckers, 2004. A rare case of oral leishmaniasis. Lancet Infect. Dis. PMID: 14720569

Velozo, D., A. Cabral, M.C.M. Ribeiro, J.O.C. Motta and I.M.C. Costa et al., 2006. Leishmaniose mucosa fatal em criança. Bras. Dermatol., 81: 255-259. DOI: $10.1590 / \mathrm{S} 0365-05962006000300008$
Virgilio, G.R. and B.R. Hale, 2004. A case of mucocutaneous leishmaniasis. Otolaryngol. Head Neck Surg., 132: 800-801.

DOI: $10.1016 /$ j.otohns.2004.09.046

Von Stebut, E., 2007. Cutaneous Leishmania infection: Progress in pathogenesis research and experimental therapy. Exp. Dermatol., 16: 340-346. DOI: $10.1111 /$ j.1600-0625.2007.00554.x 